\title{
Active Set Size Effects at HSUPA in WCDMA Systems \#
}

\author{
İsmet Çağdaş Soy ${ }^{*}{ }^{1}$, Asst. Prof. Aysel Şafak ${ }^{2}$, Hande Bakiler ${ }^{3}$ \\ Accepted $15^{\text {th }}$ August 2014 \\ DOI: 10.18100/ijamec.76437
}

\begin{abstract}
WCDMA cellular networks support soft handover, which guarantees the continuity of wireless services and enhanced communication quality. Cellular networks performance depends upon Soft handover parameters. In this paper, we have shown the effect of soft handover parameters on the performance of WCDMA cellular networks. We consider timeToTrigger1a,timeToTrigger1b, timeToTrigger1c and max active size as the Soft handover parameters. A very useful statistical measure for characterizing the performance of WCDMA cellular system is the mean active set size and soft handover region. It is shown through numerical results that above parameters have decisive effect on HSUPA, mean active set size and soft handover region and hence on the overall performance of the soft handover algorithm.
\end{abstract}

Keywords: HSUPA,WCDMA,Active Set Size, SHO, timeToTrigger

\section{Introduction}

Wide-band Code Division Multiple Access (WCDMA) based cellular standarts support soft handover, which makes smooth transition and enhanced communication quality. Soft handover offers multiple radio links to operate in parallel. Mobile users are separated by unique pseudo-random sequences and multiple data flows are transmitted simultaneously via radio interface. The User Equipment (UE) near the cell boundary is connected with more than one Base Station (BS). Consequently, in soft handover $\mathrm{UE}$ is able to get benefit from macrodiversity.

Soft handover is associated with active set and its size. The inclusion and drop of a particular BS in/from the active set is determined by the initiation trigger utilized for soft handover algorithm.

\subsection{Active Set Size in WCDMA}

Initiation trigger include, received pilot signal strength, Signal to Interference Ratio (SIR), Bit-Error Rate (BER), Energy per chip to noise power density (Ec/No). Due to the random nature of the received signal at UE, there are frequent inclusion and drop of $\mathrm{BS}(\mathrm{s})$ in the active set. Active set consists of those base stations which are connected with UE and soft handover region [15] is that region in which UE is connected with more than one base station. It is essential for properly designed soft handover algorithm [16, 17, 18, 19, 21, 22, and 23] to reduce the switching load of the system while maintaining the quality of service (QoS). In this paper, we have considered mean active set size and soft handover parameters as the metric for performance evaluation of the HSUPA throughput. It is directly related to performance of handover process with active set size.

The rest of the paper is organized as follows. Section 2 describes the system model used for real time measurements; Soft handover algorithm follows cellular layout. It is followed by

${ }^{1}$ Electrical \& Electronics Engineering Department, Institute of Science and Engineering, Başkent University, Bağlıca Campus, Ankara/Turkey * Corresponding Author: Email:21020013@baskent.edu.tr

\# This paper has been presented at the International Conference on Advanced Technology\&Sciences (ICAT'14) held in Antalya (Turkey), August 12-15, 2014. description of soft handover algorithm and numerical results are obtained, plotted and discussed in Section 3. Finally, conclusion is drawn.

\section{The System Model}

System level results are obtained by TEMS Discovery generating the statistical statements for the EUL throughput and real-time measurements are done by TEMS Investigation tool with Huawei Data card (E182) in a city. Test is done by a car and Tems logs are collected via GPS in a laptop.

\subsection{WCDMA System Model}

Received Signal Code Power (RSCP) at UE is affected by three components as follows:

(i) Path loss attenuation with respect to distance

(ii) Shadow fading

(iii) Fast fading

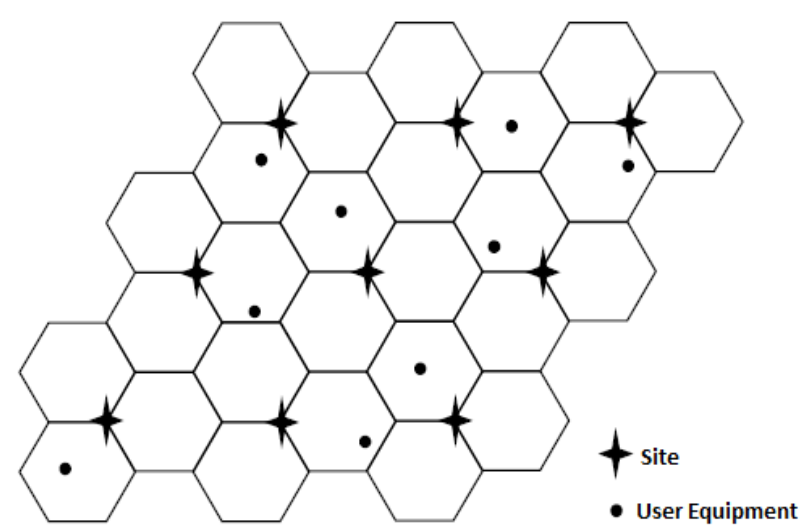

Figure 1. Network Configuration

Path loss is the deterministic component of RSCP, which can be evaluated by outdoor propagation path loss models [9 - 10]. Shadowing is caused due to the obstruction of the line of sight path between transmitter and receiver by buildings, hills, trees and foliage. Multipath fading is due to multipath reflection of a transmitted wave by objects such as houses, buildings, other man 
made structures, or natural objects such as forests surrounding the UE. It is neglected for handover initiation trigger due to its short correlation distance relative to that of shadow fading.

The UE measures RSCP from each BS. The measured value of RSCP (in $\mathrm{dBm}$ ) is the sum of two terms, one due to path loss and the other due to lognormal shadow fading. The propagation attenuation is generally modeled as the product of the $\eta^{\text {th }}$ power of distance and a log normal component representing shadow fading losses [9]. These represent slowly varying variations even for users in motion and apply to both reverse and forward links. For UE at a distance ' $\mathrm{d}$ ' from $\mathrm{BS}_{1}$, attenuation is proportional to

$\alpha(d, \zeta)=d \eta 10 \zeta / 10$

where $\zeta$ is the $\mathrm{dB}$ attenuation due to shadowing, with zero mean and standard deviation $\sigma$. Alternatively, the losses in $\mathrm{dB}$ are

$\alpha(\mathrm{d}, \zeta)[\mathrm{dB}]=10 \eta \log \mathrm{d}+\zeta$

where $\eta$ is path loss exponent. The autocorrelation function between two adjacent shadow fading samples is described by a negative exponential function as given in [11]. The measurements are averaged using a rectangular averaging window to alleviate the effect of shadow fading according to the following formula [12]. Let di denote the distance between the UE and $\mathrm{BSi}, \mathrm{i}=1,2$. Therefore, if the transmitted power of BS is Pt, the signal strength from $\mathrm{BSi}$, denoted $\mathrm{Si}$,(d) $\mathrm{i}=1,2$, can be written as [14].

$\operatorname{Si}(\mathrm{d})=\mathrm{Pt}-\alpha(\mathrm{di}, \zeta)$

$\mathrm{Si}(\mathrm{k})=(1 / \mathrm{Nw}) \Sigma \mathrm{n}=0 \mathrm{~N}-1 \mathrm{Si}(\mathrm{k}-\mathrm{n}) \mathrm{Wn}$

where; $\mathrm{Si}$ is the averaged signal strength and $\mathrm{Si}$ is the signal strength before averaging process. $\mathrm{Wn}$ is the weight assigned to the sample taken at the end of $(\mathrm{k}-\mathrm{n})$ th interval. $\mathrm{N}$ is the number of samples in the averaging window

$$
\mathrm{N}_{\mathrm{W}}=\sum_{n=0}^{N-1} W_{n} .
$$

Table 1. Simulation Parameter

\begin{tabular}{|c|c|}
\hline $\mathrm{D}=400 \mathrm{~km}$ & Drive distance per test \\
\hline $\mathrm{UL}=10 \mathrm{Mhz}$ & UL Bandwidth \\
\hline $\mathrm{ds}=1 \mathrm{~s}$ & Sampling time \\
\hline $\begin{array}{c}\mathrm{CPICH}=340 \\
\mathrm{dBm}\end{array}$ & Base station transmitter power \\
\hline $\mathrm{N}=126$ & Site count in the city \\
\hline
\end{tabular}

\section{Soft Handover Algorithm}

Based on the Ec/Io measurements of the set of cells monitored, the mobile station decides which of three basic actions to perform; it is possible to add, remove or replace a NodeB in the active cell. These tasks are respectively called Radio Link Addition and Radio Link Removal, while the latter is Combined Radio Link Addition and Removal. The example below is directly taken from the original 3GPP specifications. Discussing this scenario gives a good insight into the algorithm itself and forms an introduction to the illustrating simulations included in the next paragraph. This scenario can be based on a user following a trajectory as shown below. Delays in handovers can cause a user to penetrate deeply in an adjacent cell and generate harmful interference before the cell is added to the active cell.
Figure shows how the pilot signal strengths of the different cells evolve in time.

When the measurement values satisfy the following formulas, the UE deems that a primary pilot channel has entered the reporting range:

Path Loss:

10Log $M_{\text {new }}<\mathrm{W} \times 10 \log \left(\sum_{j=1}^{N a} M i\right)+(1-\mathrm{W}) \times 10 \log M_{\text {Best }}+\left(\mathrm{R}-\mathrm{H}_{1 \mathrm{a} / 2}\right)$,

Other measurement values:

$10 \log M_{\text {new }}<W \times 10 \log \left(\sum_{j=1}^{N a} M i\right)+(1-W) \times 10 \log M_{\text {Best }}-\left(R-\mathrm{H}_{1 a / 2}\right)$,

Where,

Mnew is the measurement result of the cell that has entered the reporting range

$\mathrm{Mi}$ is the measurement result of the cells in the active set

$\mathrm{Na}$ is the number of cells in the current active set

MBest is the measurement result of the best cell in the current active set

$\mathrm{W}$ is the weight factor

$\mathrm{R}$ is the reporting range. With the signal strength as an example, $\mathrm{R}$ equals to the signal strength of the best cell in the current active set minus a value

$\mathrm{H} 1 \mathrm{a}$ is the hysteresis value of event $1 \mathrm{~A}$

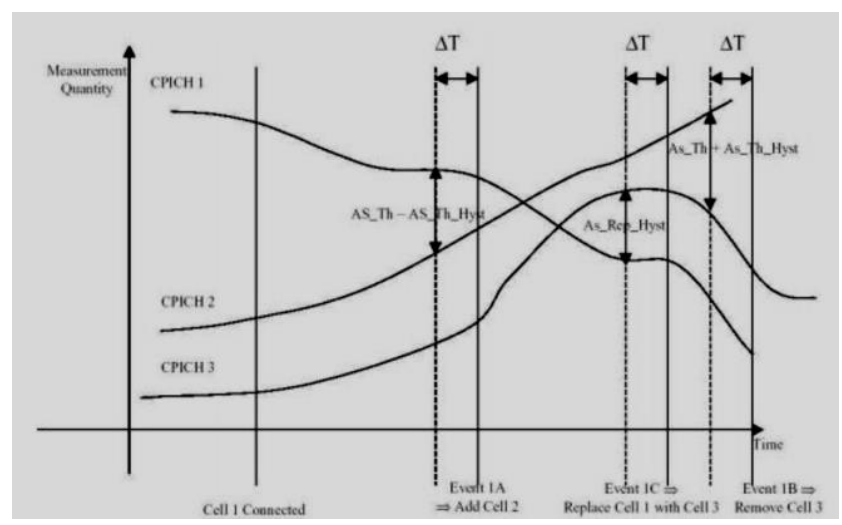

Figure 2. WCDMA handover algorithm

In explanation of soft handover algorithm, the following terms are crucial, and have to be defined:

Active set contains the list of cells, having the connection with MS, in other words, the cells, which are in soft handover connection.

Monitored set contains the list of cells, which pilot channels power is not high enough to be added to the active set, or active set is already full.

Detected set contains the list of cells, which are not defined as neighbour to the AS cells. Detected cells pilot-channels power can be high enough(must defined neighbour) or not.

At the start of the scenario the user is connected to cell number 1 which has the strongest pilot signal. Due to the user moving or to slow fading the perception of the signal strengths to the mobile user can change and following actions are taken:

- Event 1A: cell 2 is added

- Event 1B: cell 1 is replaced with cell 3

- Event 1C: Cell 3 is removed from the active set

\subsection{EUL - Enhanced Uplink:}

HSUPA uses an uplink enhanced dedicated channel (E-DCH) on which it employs link adaptation methods similar to those employed by High-Speed Downlink Packet Access HSDPA, 
namely:

Shorter Transmission Time Interval enabling faster link adaptation;

HARQ (hybrid ARQ) with incremental redundancy making retransmissions more effective.

Similarly to HSDPA, HSUPA uses a packet scheduler, but it operates on a request-grant principle where the UEs request a permission to send data and the scheduler decides when and how many UEs will be allowed to do so. A request for transmission contains data about the state of the transmission buffer and the queue at the UE and its available power margin. However, unlike HSDPA, uplink transmissions are not orthogonal to each other. In addition to this scheduled mode of transmission the standards also allows a self-initiated transmission mode from the UEs, denoted non-scheduled. The non-scheduled mode can, for example, be used for VoIP services for which even the reduced TTI and the Node B based scheduler will not be able to provide the very short delay time and constant bandwidth required.

Each MAC-d flow (i.e. QoS flow) is configured to use either scheduled or non-scheduled modes; the UE adjusts the data rate for scheduled and non-scheduled flows independently. The maximum data rate of each non-scheduled flow is configured at call setup, and typically not changed frequently. The power used by the scheduled flows is controlled dynamically by the Node B through absolute grant (consisting of an actual value) and relative grant (consisting of a single up/down bit) messages.

At the Physical Layer, HSUPA introduces new channels EAGCH (Absolute Grant Channel), E-RGCH (Relative Grant Channel), F-DPCH (Fractional-DPCH), E-HICH (E-DCH Hybrid ARQ Indicator Channel), E-DPCCH (E-DCH Dedicated Physical Control Channel) and E-DPDCH (E-DCH Dedicated Physical Data Channel).

E-DPDCH is used to carry the E-DCH Transport Channel; and E$\mathrm{DPCCH}$ is used to carry the control information associated with the E-DCH.

As shown in Table 2 below all UE categories support maximum data rates.

Table 2. UE HSUPA Categories

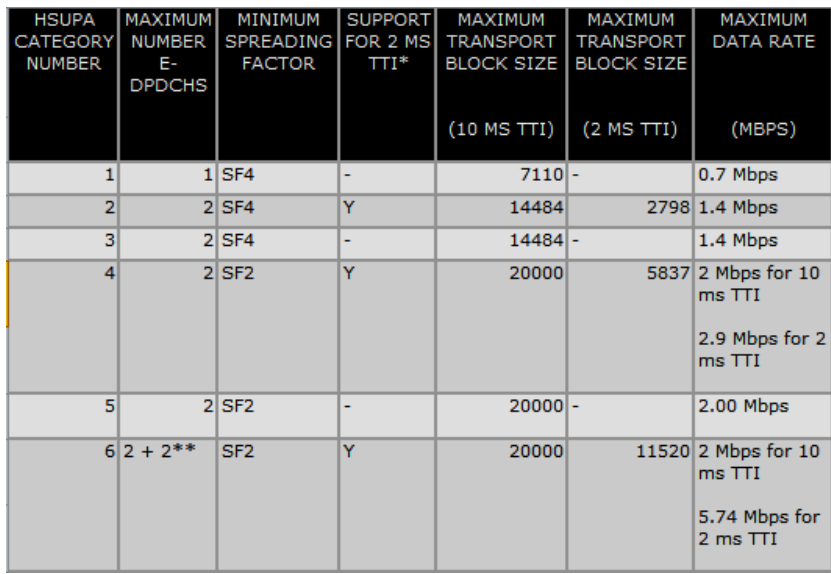

\subsection{EUL Throughput Measurement Analyse:}

In this section, mean active set size, soft handover and EUL throughput has been measured as the function of different system parameters and characteristic parameters of radio propagation environment. Numerical results for this performance metric are obtained via TEMS Investigation for the system parameters indicated in Table 1. System measurement is performed TEMS Investigation 15.1 and analyzed by TEMS Discovery 10.1. To obtain different active set size and EUL throughput for each system parameter setting, 3 drive tests are performed in the same city center.

Figure $3 \mathrm{a} \& 3 \mathrm{~b}$ show the effect of AS Size on the SIR and EUL TTI usage. $2 \mathrm{~ms}$ TTI usage decreased when Active set size increases. When the UE start to connect more cells upload direction, capacity usage increased so that $10 \mathrm{~ms}$ TTI usage increased. For both 3 drive test SIR values or very similar to each of them.

Figure 4a shows the EUL Throughput Distribution for all AS Size in the city. That figure shows that distribution percentage of all band. Figure $4 b$ shows the Median throughput for each active set size measurements. If we tell the for same SIR values, EUL tput increases with the $2 \mathrm{~ms}$ TTI usage.

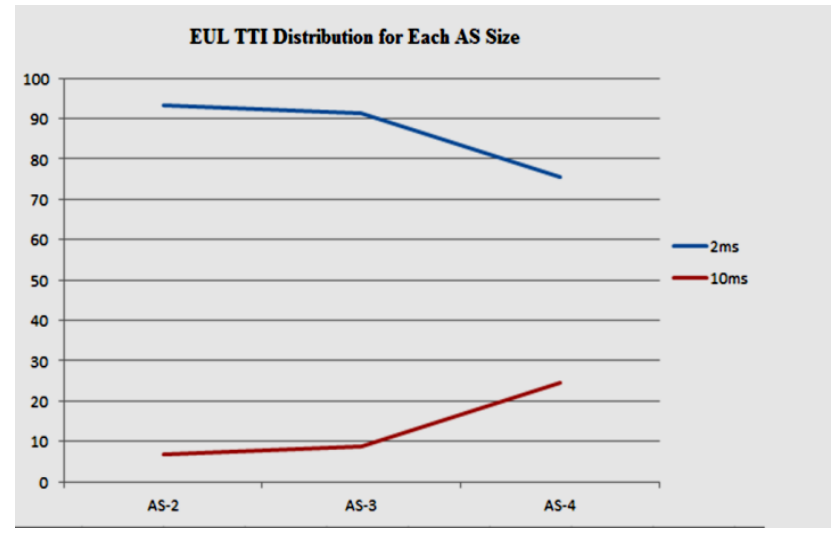

Figure 3a. Effect of AS size for EUL TTI Usage.

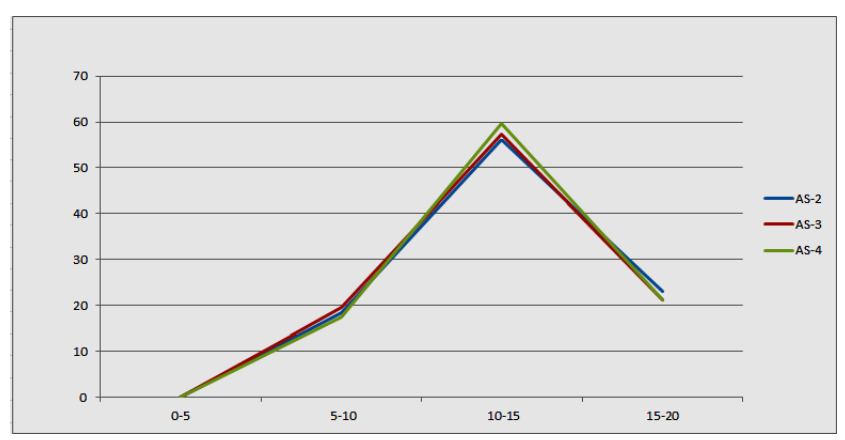

Figure 3b. Effect of AS size for SIR

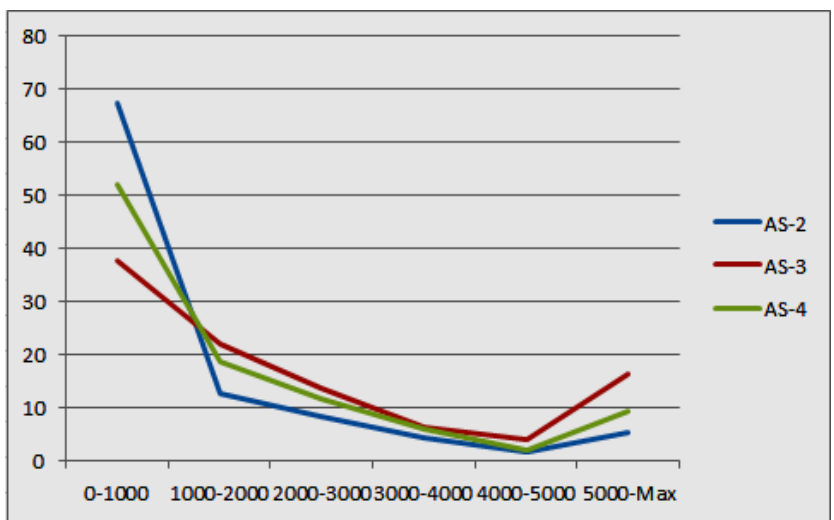

Figure 4a. Effect of AS size for EUL Distribution 


\begin{tabular}{|c|c|}
\hline EUL Median Tput(kbps) & kbps \\
\hline AS-2 & 2034.7 \\
\hline AS-3 & 1781.1 \\
\hline AS-4 & 1579 \\
\hline
\end{tabular}

Figure 4b. EUL Median Tput

\section{Conclusion}

In this paper, we have tried to understand the impact of Active Set Size on EUL performance, which was measured in a city with TEMS Investigation tool. Measurement results show that active set size setting has a decisive effect on the EUL performance. By careful tuning and setting appropriate values for the active set size parameter, a higher system performance can be achieved.

\section{References}

[1] K. S. Gilhousen, I. M. Jacobs, R. Padovani, L. Weaver. Increased Capacity using CDMA for Mobile Communications, IEEE Journal on Selected Areas in Communications, Vol. 8, May 1990, pp. 503-514.

[2] A. J. Viterbi, A. M. Viterbi, K. S. Gilhousen, E. Zehavi. Soft Handoff Extends Cell Coverage and Increases Reverse Link Capacity, IEEE Journal on Selected Areas in Communications, Vol. 12, October 1998, pp. 1281-1287.

[3] N. Jhang, J. M. Holtzman. Analysis of CDMA SoftHandoff Algorithm, IEEE Transactions on Vehicular Technology, Vol. 47, May 1998, pp. 710-714.

[4] B. Homnan, V. Kunsriruksakul, W. Benjapolakul. A Comparative Evaluation of Soft Hnadoff between S-95A and IS-95B/cdma2000, Proc. IEEE Vehicular Technology, 2000, pp. 34-37.

[5] V. Vassiliou, J. Antoniu, A. Pitsillides, G. Hadjipollas. Simulating Soft Handover and Power Control for Enhanced UMTS, IEEE 16th International Symposium on Personal, Indoor and Mobile Radio Communications, 2005, pp. 16461651.

[6] D. Zhang, G, Wei, J. Zhu. Performance of Hard and Soft Handover for CDMA Systems, IEEE Vehicular Technology Conference 2002, pp. 1143-1147.

[7] J. Reig. Capacity Analysis in Downlink WCDMA Systems Using Soft Hanodver Techniques With SIR-Based Power Control and Site Selection Diversity Transmission, IEEE Transactions on Vehicular Technology, Vol. 55, July 2006, pp. 1362-1372.

[8] A. E. Leu, B. L. Mark. Discrete-time Analysis of Soft Handoff in CDMA Cellular Networks, Proc. IEEE Vehicular Technology Conference, 2002, pp. 3222-3226.

[9] V. K. Garg, J. E. Wilkes. Principles and Applications of GSM, Prentice Hall PTR, Upper Saddle River, NJ 07458.

[10] K. Feher. Wireless Digital Communications, Modulation and Spread Spectrum Applications, Prentice-Hall of India,
New Delhi-2001.

[11] V. M. Gudmundson. Correlation Model for Shadow Fading in Mobile Radio Communication System, Electronics Letters., vol.27, November 1991, pp.2145-2146.

[12] G. E. Corazza D. Giancristofaso, F. Santucci. Characterization of Handover Initiation in Cellular Mobile Radio Networks, IEEE Technology Conference, 1994, pp. 1869-1872.

[13] N.P.Singh, Brahmjit Singh. Effects of Soft Handover Margin under various Radio propagation parameters in CDMA Cellular Networks, IEEE conference on WCSN2007, pp 47-50.

[14] Brahmjit Singh. An improved handover Algorithm based on signal strength plus distance for interoperability in mobile cellular networks, Wireless Personal Communication (2007), 43:879-887.

[15] Xinbing Wang, Shiguang Xie, and Xiuwen Hu. Recursive Analysis for Soft Handoff Schemes in CDMA Cellular Systems, IEEE Transaction on Wireless Communications, Vol.8, No. 3, March 2009.

[16] Qualcomm Corporation. Diversity-Handover method and performance, ETSI SMG2 Wideband CDMA Concept GroupAlpha Meeting, Stockholm, Sweden, Sept. 1997.

[17] EIA/TIA/IS-95A. Mobile Station-Base Station Compatibility Standard for Dual-Mode Wideband Spread Spectrum Cellular System, Telecommunication Industry Association, Washington CD, May 1995.

[18] J. Laiho-Steffens, M. Jasberg, K. Sipila, A. Wacker and A. Kangas. Comparison of three diversity handover algorithms by using measured propagation data, Proceedings of VTC'99 Spring, Houston, TX, May 1999, pp. 1370-1374.

[19] Universal Mobile Telecommunications System (UMTS); Radio Resource Management Strategies, (3GPP TR 25.922 version 5.0.0 Release 5)

[20] R. P. Narrainen and F. Takawira. Performance analysis of soft handoff in CDMA cellular networks, Vehicular Technology, IEEE Transactions on, Vol. 50, Issue. 6, Nov. 2001, pp. 1507-1517.

[21] X. Yang, S. Ghaheri-Niri and R. Tafazolli. Performance of power triggered and Ec/NO- triggered soft handover algorithms for UTRA, 3G Mobile Communication Technologies, Conference Publication, No. 477, 2000, pp. 7-10.

[22] X. Yang, S. Ghaheri-Niri and R. Tafazolli. Evaluation of soft handover algorithms for UMTS, Personal, Indoor and Mobile Radio Communications, 2000 11th IEEE International Symposium on, Vol. 2, 2000, pp. 772-776.

[23] S. S. Wang, S. Sridsha and M. Green. Adaptive soft handoff method using mobile location information, Vehicular Technology Conference, 2002, VTC Spring 2002. IEEE 55th, vol. 4, 2002, pp. 1936-1940.

[24] N. P. Singh, Brahmjit Singh. Effect of soft handover parameters on CDMA cellular networks, Journal of Theoretical and Applied Information Technology, 2005, pp. 110-115. 